\title{
Surgically-Treated Locoregionally Advanced Hypopharyngeal Cancer: Outcomes
}

\author{
Jorge Rodrigues $^{1,2}$ Eduardo Breda ${ }^{3}$ Eurico Monteiro ${ }^{3}$ \\ ${ }^{1}$ Department of Otorhinolaringology, Hospital da Senhora da Oliveira \\ Guimarães EPE, Creixomil, Guimarães, Portugal \\ 2 Department of Anatomy, University of Porto, Portugal \\ ${ }^{3}$ Department of Otorhinolaringology, Instituto Português de \\ Oncologia do Porto Francisco Gentil EPE, Porto, Portugal

\begin{abstract}
Address for correspondence Jorge Rodrigues, MD, Department of Otorhinolaringology, Hospital da Senhora da Oliveira Guimarães EPE, Creixomil, Guimarães 4835-044, Portugal (e-mail: jorge.rods@hotmail.com).
\end{abstract}

Int Arch Otorhinolaryngol 2018;22:443-448.

\begin{abstract}
Keywords

- laryngeal cancer

- laryngectomy

- hypopharyngeal cancer

- laryngeal neoplasms

- hypopharyngeal neoplasms

Introduction Hypopharyngeal tumors are head and neck malignancies associated with a great mortality rate, and the treatment of advanced lesions constitutes a challenging problem. Pharyngolaryngectomy continues to be the gold standard treatment modality for locally-advanced diseases, and it is currently used as the primary treatment or in cases of relapse after an organ preservation strategy.

Objective This study aims to compare the survival rates of patients with advanced hypopharyngeal tumors treated with pharyngolaryngectomy as a primary or salvage option, and identify possible prognostic factors.

Methods All patients with advanced hypopharyngeal squamous cell carcinomas who performed pharyngolaryngectomy between 2007 and 2014 were reviewed retrospectively.

Results A total of 87 patients fulfilled the aforementioned criteria, and the sample had a mean age of 57.2 years and a male predominance of $43: 1$. The tumors were located in the pyriform sinus walls (81 tumors), in the posterior pharyngeal wall (4 tumors) and in the postcricoid region ( 2 tumors). A total of 60 patients underwent surgery as the primary treatment option, and 27 were submitted to salvage pharyngolaryngectomy after a previous treatment with chemoradiotherapy or radiotherapy. The 5 -year overall survival was of $25.9 \%$, the 5 -year disease-free survival was of $24.2 \%$, and the disease-specific survival was of $29.5 \%$.

Conclusion The patients treated with pharyngolaryngectomy as the primary option revealed a better 5 -year-disease free survival than the patients who underwent the salvage surgery ( $35.8 \%$ versus $11.7 \%$ respectively; $p<0.05$ ). The histopathological criteria of capsular rupture of the lymph nodes (30.1\% versus $19.8 \%$ respectively for the primary and salvage groups; $p<0.05)$ and vascular invasion $(30.5 \%$ versus $22.5 \%$ respectively; $p<0.05$ ) reduced the 5 -year disease-free survival. Pharyngolaryngectomy as the primary intent revealed a lower local recurrence rate than the salvage surgery ( $40.6 \%$ versus $83.3 \%$ respectively; $p<0.05$ ).
\end{abstract}

received

September 29, 2017

accepted

February 5, 2018

published online

July 5, 2018
DOI https://doi.org/

10.1055/s-0038-1641562.

ISSN 1809-9777.
Copyright $(2018$ by Thieme Revinter

Publicações Ltda, Rio de Janeiro, Brazil
License terms

(ㄷ) (i) $\ominus$ (\$) 


\section{Introduction}

Head and neck cancer represents the fifth most common cancer worldwide, and comprises a heterogeneous group of tumors with different behaviors and prognosis. ${ }^{1,2}$ More than $90 \%$ of these tumors are squamous cell carcinomas, and the vast majority occurs in individuals with a history of exposure to carcinogens, such as tobacco and alcohol. ${ }^{3,4}$

Hypopharynx tumors contribute to $\sim 7 \%$ of upper aerodigestive tract tumors, affecting $\sim 14.8$ per 100,000 habitants in Southern Europe ${ }^{5-7}$ The mean age at presentation is usually $\sim 65$ years, with an apparently tendency to an earlier onset. ${ }^{5}$ Lesions of the pyriform sinus and posterior pharyngeal walls tend to be more predominant among the male population, while postcricoid lesions seem to demonstrate a female preponderance of $1.5: 1{ }^{8}$

Upon presentation, more than $75 \%$ of patients exhibit the disease in advanced stages, that is, with tumors with large volumes and lymph node metastasis. ${ }^{9}$ Moreover, $\sim 60 \%$ of the patients without clinically-detectable neck diseases present micrometastases in elective neck dissections. ${ }^{9}$ Distant metastases develop in $\sim 25 \%$ of the patients, and the lungs, the liver and the bones are the most affected sites. ${ }^{10,11}$

The primary goal of the treatment is to achieve the highest locoregional control with the least functional disability, preserving regular breathing, deglutition and phonation whenever possible. ${ }^{12}$ Early-stage tumors are generally managed with a single-modality therapy, either partial surgery or radiotherapy (RT). ${ }^{13}$ There is no consensus about the best option to achieve better survival rates and less morbidity in cases of advanced tumors. These tumors are usually treated with a combined-modality therapy, either primary pharyngolaryngectomy (PL) followed by chemoradiotherapy (CRT), or primary CRT with salvage surgery when needed. ${ }^{14,15}$ Recently, organ preservation strategies became more and more widely used. However, a significant number of patients included in these strategies need PL as salvage option, but the possible impact on survival remains unclear. ${ }^{12-16}$

The prognosis of hypopharyngeal cancer remains poor, with a 5 -year overall survival (OS) rarely exceeding $35 \%{ }^{7}$ While some studies report a 5-year OS 70\% in early stages, most cases are advanced, and survival drops to $25 \%^{7,14}$ Hoffman et al analyzed a large number of patients, the majority in stages III or IV, and identified a disease-specific survival (DSS) rate of $33.4 \% .^{14}$

The objective of the present review is to analyze the outcomes of patients submitted to PL either as the primary or salvage option, according to stage. Additionally, it is also our aim to identify the main cause of death and any histopathological prognostic factors that may have an impact on survival.

\section{Materials and Methods}

A retrospective study was performed including patients with advanced hypopharyngeal tumors treated with curative intent, between 2007 and 2014, in an oncological tertiary center. Approval of the Medical Ethics Committee was obtained (CES.155/015). According to the National Comprehensive Cancer Network (NCCN) guidelines, patients with T3 tumors were selected to undergo CRT, and to those with T4a tumors, radical surgery with the multidisciplinary oncological team was proposed. Radical surgery was also proposed to the patients with T3 and T4a tumors who refused to undergo CRT, and to those who presented contraindications to CRT due to severe comorbidities. ${ }^{17}$ - Table 1 shows the treatment selection. Patients with laryngeal or esophageal tumors with extension to the hypopharynx, and patients with other synchronous malignancies were excluded, as well as patients with incomplete clinical records. Age and gender, tumor, node and metastasis (TNM) staging according to the Cancer Staging Manual of the American Joint Committee on Cancer (AJCC, 2002), ${ }^{18}$ treatment options, recurrence, global and specific survival and cause of death were analyzed. ${ }^{17,18}$ Furthermore, histopathological charts were analyzed for eventual prognostic factors such as positive margins, capsular rupture of lymph nodes, and perineural and vascular invasion. The cut-off point for clear resection margins was a minimum distance of $5 \mathrm{~mm}$ from the invasive front tumor.

Local recurrence was defined as an invasive carcinoma developing 6 months after the conclusion of the curative treatment. Regional and distal recurrences were defined as the presence of lesions with the same histological type in regional lymph nodes or distant sites respectively, after the completion of the initial treatment.

All patients were assessed for 5-year OS, disease-free survival (DFS), and DSS, with respect to tumor location, staging, type of primary treatment, histopathological findings and adjuvant therapy. The survival rates were measured using the time from the end of the treatment.

Survival curves were created using the Kaplan-Meier method, and significant differences among the actuarial curves were tested by Log-rank test. The statistical analysis was performed using the Statistical Package for the Social Sciences (SPSS, IBM SPSS Statistics for Windows, IBM Corp., Armonk, NY, US), version 20.0. Values of $p<0.05$ were considered statistically significant.

\section{Results}

A total of 87 patients satisfied the inclusion criteria. All of them had CT3 or cT4 hypopharyngeal tumors. Regarding gender, $85(97.7 \%)$ patients were men, and $2(2.3 \%)$ were women, resulting in a male/female ratio of $43: 1$. The mean

Table 1 Treatment selection

\begin{tabular}{|l|l|l|l|l|}
\hline & RT & CRT & Radical surgery & Total \\
\hline T3 & 3 & 24 & $11\left(^{*}\right)$ & 38 \\
\hline T4 & 0 & 0 & 49 & 49 \\
\hline total & 3 & 24 & 60 & 87 \\
\hline
\end{tabular}

Abbreviations: CRT, chemoradiotherapy; RT, radiotherapy. Note: * A total of 4 patients refused surgery, and 7 patients presented contraindication to CRT. 
Table 2 Characteristics of the patients

\begin{tabular}{|l|l|l|l|}
\hline \multirow{2}{*}{ Total } & & N & $\%$ \\
\hline \multirow{3}{*}{ Age } & & 87 & $100 \%$ \\
\hline \multirow{3}{*}{ Gender } & $\leq 59$ & 53 & $60.9 \%$ \\
\cline { 2 - 4 } & $>59$ & 34 & $39.1 \%$ \\
\hline \multirow{3}{*}{ Management } & Male & 85 & $97.7 \%$ \\
\cline { 2 - 4 } & Female & 2 & $2.3 \%$ \\
\hline \multirow{2}{*}{ Previous treatment } & Primary & 60 & $69.0 \%$ \\
\cline { 2 - 4 } & Salvage & 27 & $31.0 \%$ \\
\cline { 2 - 4 } & Radiotherapy & 3 & $11.1 \%$ \\
\cline { 2 - 4 } & Chemoradiotherapy & 24 & $88.9 \%$ \\
\hline
\end{tabular}

age at presentation was 57.2 years (standard deviation $[S D]=8.1$ ), ranging from 38 to 76 years old. Concerning the anatomical site, 81 tumors (93\%) arose from the pyriform sinus walls, 4 (4.6\%) from the posterior pharyngeal wall, and $2(2.3 \%)$ were originated in the postcricoid region.

Surgery as the primary treatment, including PL and bilateral neck dissection, was performed in 60 patients. After a previous organ preservation treatment with CRT or RT, 27 patients were submitted to salvage PL. Adjuvant RT or CRT was performed in all patients with positive or close surgical margins. To the patients with other adverse features, such as primary pT4, N2 or N3 nodal disease, perineural invasion or vascular embolism, CRT (49/60) or RT alone (2/60), depending on the patients' general conditions and comorbidities, were indicated. - Table $\mathbf{2}$ summarizes the management of the patients.

Among all 87 patients, 38 (44\%) presented with cT3 tumors, and 49 (56\%) with cT4. However, after the histopathological surgical specimen analysis, 67 patients $(77 \% ; 61$ patients with tumors in the pyriform sinus, 4 in the posterior pharyngeal wall, and 2 in the postcricoid region) had pT4 tumors, resulting in an estimated understaging of $21 \%$ (-Table 3). Similarly, 38 (63\%) out of the 60 patients submitted to primary PL were clinically classified as $\mathrm{cN} 2$ or cN3. Nevertheless, after the histopathological examination, $90 \%$ $(54 / 60)$ of these tumors were pN2 or pN3, resulting in an understaging of $\sim 27 \%$.

The histopathological evaluation of the margins in primary specimens showed free margins in $71.3 \%$ (62/87) of them. A total of 67 (77\%) patients presented extra-organ

Table 3 Tumor details classified by primary site and T stage

\begin{tabular}{|l|l|l|l|l|l|}
\hline & cT3 & pT3 & cT4 & pT4 & Total \\
\hline Hypopharynx & 38 & 20 & 49 & 67 & 87 \\
\hline Pyriform sinus & 33 & 20 & 48 & 61 & 81 \\
\hline Posterior pharyngeal wall & 3 & 0 & 1 & 4 & 4 \\
\hline Postcricoid region & 2 & 0 & 0 & 2 & 2 \\
\hline
\end{tabular}

Abbreviations: c, clinical stage; $p$, pathological stage. invasion of adjacent structures, mostly to the larynx. Perineural and vascular invasion was observed in 52\% (45/87) and 56\% (49/87) of tumors respectively. The histopathological evaluation of the margins in primary specimens showed free margins in 71.3\% (62/87) of them. Capsular rupture was demonstrated in $46 \%$ of the 68 patients presenting neck disease. No significant difference between the primary or salvage surgery groups was obtained when comparing the rate of close or positive margins (28.3\% versus $29.6 \%$ respectively; $p>0.05$ ), perineural invasion (53.3\% versus $48.1 \%$ respectively; $p>0.05$ ), and vascular invasion (58.3\% versus $51.9 \%$ respectively; $p>0.05)$.

The 5-year OS was of $25.9 \%$ (SD = 7.2\%); the 5-year DFS was of $24.2 \%$ (SD $=6.4 \%$ ); and the 5 -year DSS was of $29.5 \%$ $(\mathrm{SD}=0.8 \%$ ) (-Fig. 1). The location of the tumor did not statistically modify the OS, DFS or DSS. The 5-year OS was of $36.1 \%$ in patients treated with primary $\mathrm{PL}$, and of $12.2 \%$ in patients who performed surgery as a salvage procedure $(p<0.05)$. The 5 -year DFS was of $35.8 \%$ in patients treated with primary pharyngolaryngectomy, and of $11.7 \%$ in patients who underwent surgery as a salvage procedure $(p<0.05$ ) (-Fig. 2). Finally, the 5-year DSS survival was of $36.2 \%$ in patients treated with primary PL, and of $16.1 \%$ in patients who underwent surgery as a salvage procedure $(p<0.05)$.

Concerning the histopathological analysis, only capsular rupture of the lymph nodes (DFS: $30.1 \%$ versus $19.8 \%$ respectively for the primary and salvage surgery groups; $p=0.012$ ) and vascular invasion (DFS: $30.5 \%$ versus $22.5 \%$ respectively; $p=0.001)$ revealed statistical significance (-Table 4).

During the follow-up period, $26 \%$ of the patients developed local recurrence, 9\% developed regional relapse, and $14 \%$ presented with distant metastases. Greater rates of local recurrence $(p<0.05)$ were associated with pT4 tumors and extra-organ spread. Distant metastases were significantly higher in pT4 and pN2-N3 tumors, and in patients with capsular rupture of the lymph nodes.

Local recurrence was less often observed in patients submitted to PL as a primary intent than in those submitted to the salvage surgery ( $40.6 \%$ versus $83.3 \%$ respectively; $p<0.05$ ), without difference according to the origin of the tumor.

\section{Discussion}

The natural history of patients with squamous cell carcinoma of the hypopharynx seems to be still poorly understood. Hypopharyngeal cancer has been associated with exposure to carcinogens in a harmful lifestyle. ${ }^{3}$ These unhealthy behaviors appear to predominate in Southern Europe, where a higher incidence is also reported. 2,5,6,19

In the present study, which included 87 patients, the mean age of presentation and the gender ratio seem to be similar to those mentioned in the literature. ${ }^{7,19}$ Male predominance appears to be related with the higher alcohol consumption among men; however, this relationship could not be rigorously evaluated in this revision., 

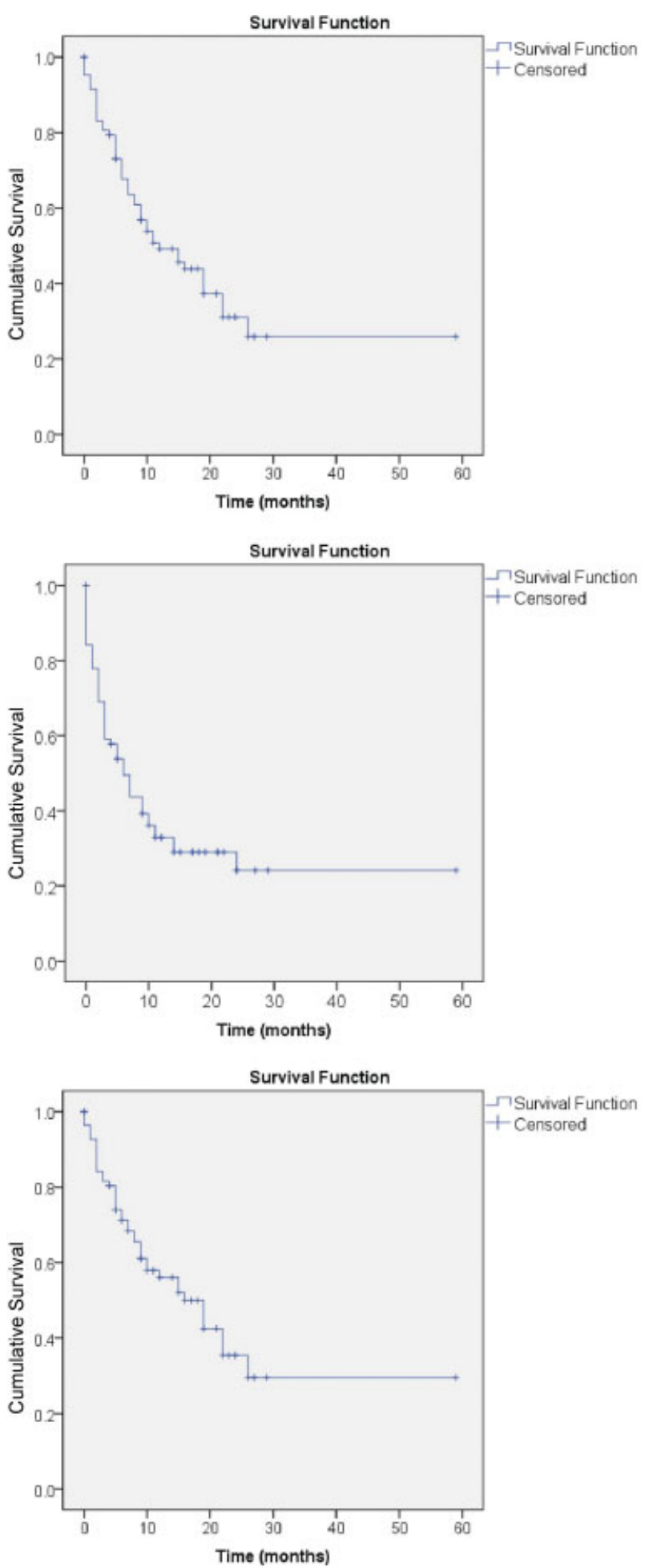

Fig. 1 Five-year OS, DFS and DSS of the patients with hypopharyngeal carcinoma.

A total of 81 (93\%) of the selected cases had tumors that arose from the pyriform sinus walls, followed by $4.6 \%(4 / 87)$ in the posterior pharyngeal wall, and $2.3 \%(2 / 87)$ in the postcricoid region. Similarly, Kirchner ${ }^{19}$ analyzed 152 hypopharynx tumors, and verified that $86 \%$ of them arose in the pyriform sinus walls, $9 \%$ occurred in the posterior pharyngeal wall, and $5 \%$ originated in the postcricoid region, with a

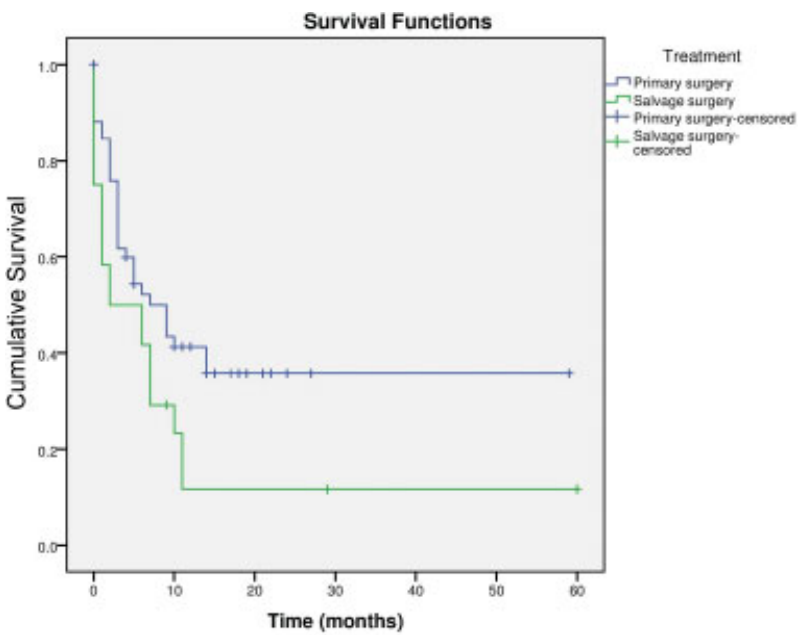

Fig. 2 Five-year DFS of the patients with hypopharyngeal carcinoma according to the type of management.

female predominance in this last site. ${ }^{8,19}$ Additionally, Berrino and Gatta, in a European cancer registry review, observed a higher proportion of pyriform sinus malignant tumors in France than in the United Kingdom or Sweden. ${ }^{20}$ The true reason for this subsite variation among countries remains unclear. ${ }^{20}$ Regarding origin, posterior pharyngeal wall lesions appear to present slightly worse outcomes. ${ }^{8}$ The small number of patients with this tumor in this location in the present series did not enable us to draw any conclusions about this matter.

Table 4 Impact of histopathological features in 5-year DFS in hypopharyngeal cancer

\begin{tabular}{|l|l|l|}
\hline & 5-year DFS & $p$ \\
\hline Primary & $35.8 \%$ & 0.033 \\
\hline Salvage & $11.7 \%$ & \\
\hline pT3 & $40.9 \%$ & 0.137 \\
\hline pT4 & $20.9 \%$ & \\
\hline pN0-N1 & $35.7 \%$ & 0.879 \\
\hline pN2-N3 & $27.6 \%$ & \\
\hline$>5$ mm free margin & $35.4 \%$ & 0.770 \\
\hline Close or positive margin & $21.4 \%$ & \\
\hline Extra-organ spread (-) & $26.3 \%$ & 0.690 \\
\hline Extra-organ spread (+) & $15.5 \%$ & \\
\hline Capsular rupture of lymph node (-) & $30.1 \%$ & $\mathbf{0 . 0 1 2}$ \\
\hline Capsular rupture of lymph node $(+)$ & $19.8 \%$ & \\
\hline Perineural invasion (-) & $30.3 \%$ & 0.631 \\
\hline Perineural invasion (+) & $21.8 \%$ & \\
\hline Vascular invasion (-) & $30.5 \%$ & $\mathbf{0 . 0 0 1}$ \\
\hline Vascular invasion (+) & $22.5 \%$ & \\
\hline
\end{tabular}

Abbreviation: DFS, disease-free survival. 
According to the consulted bibliography, different strategies may be adopted for hypopharyngeal cancer management, and there is no consensus about which is the best strategy to reach higher survival rates and less morbidity. $^{21-23}$ The evolution of RT devices, the selection of new chemotherapy agents, and the introduction of new surgical procedures, such as transoral laser and/or robotics enabled the emergence of organ preservation strategies as admissible options in cases of early stages. ${ }^{21,22}$ However, in cases of advanced stages, these strategies present several limitations, and functional preservation of the organ/region is not always achieved. $^{23,24}$

The present series also revealed that the rate of close or positive margins and of perineural and vascular invasion were similar in patients who underwent primary versus salvage PL, probably because the majority of the cases presented locoregionally advanced diseases. ${ }^{25,26}$

Advanced stage at presentation may be the reason why hypopharyngeal tumors are associated with the poorest survival rates among all squamous cell carcinomas of the upper aerodigestive tract, with an OS that, in the majority of papers, does not exceed $35 \%$ after 5 years. $^{7}$

In the present study, which comprised patients with advanced-stage tumors, the estimated OS was of $25.9 \%$. Hoffman et al published a review of 2,939 cases, including $75 \%$ in stages III-IV, and reported a 5 -year DSS of $\sim 33.4 \% .{ }^{27}$ In the same study, according to stage, the 5-year DFS was of $41.8 \%$ for stage III, and of $22 \%$ for stage IV, and the cases were treated with different modalities: isolated surgery (50.4\%); surgery combined with irradiation (48\%); and irradiation alone (25.8\%). ${ }^{27}$

In the present paper, the 5-year DFS was of $24.2 \%$, and the DSS was of 29.5\%. Comparing these results with other series, we verified similar survival rates, supporting the idea that hypopharyngeal tumors continue to have an unsatisfactory prognosis that has not changed appreciably over the past decades. $^{5-7,27}$

As shown in - Fig. 2, the patients treated with PL as a primary option revealed a better 5-year DFS than the patients who performed surgery as a salvage procedure (35.8\% versus $11.7 \%$ respectively; $p<0.05$ ). The rate of unsafe margins and perineural or vascular permeation is similar in both primary and salvage surgeries; therefore this statistically significant difference on survival needs clarification. An explanation could be that some delay on the diagnosis of tumor recurrence in organ preservation patients could result in the probable deferral of treatment relapse, consequently worsening the prognosis. Moreover, fibrosis and edema result in additional morbidity in cases of salvage surgeries, increased susceptibility to complications, and poor survival rates. ${ }^{12,15,24,27}$ Our findings are in line with those reported by Hoffman et al, who reported higher survival rates in patients initially treated with surgery. ${ }^{27}$ According to statistics published by Siegel et al, advanced laryngeal tumors showed a decrease in survival in the last years, which is apparently related with higher locoregional recurrence in patients treated with organ preservation strategies. ${ }^{15}$ In Europe, studies have not been able to clarify this difference when applied to pharyngolaryngeal tumors. $^{25}$

Several other aspects have also been identified in the literature as affecting the survival rates of these patients, namely the location and extension of the lesions, the general condition of the patients, and the treatment options. The pathological extent of the disease and the histological characteristics appear to be determinant regarding the survival outcomes. Johnson et al reported a relationship between tumor volume and local control rates. ${ }^{28}$ The pattern of tumor extension and extra-organ spread was widely investigated, revealing a negative impact on survival. ${ }^{28}$

Concerning the histopathological criteria, some characteristics are reported as predictive of prognosis, such as positive surgical margins, vascular invasion, neural permeation, regional metastases, and extracapsular rupture of the lymph nodes. $^{25,26}$ Complete excision of the local disease remains a crucial principle in oncologic surgery. Frozen section procedures are a useful aid to examine tissue samples while the surgery is taking place, minimizing the possibility of leaving a residual tumor. The local recurrence rate is clearly increased if the excision margins are positive, and that decreases the survival rate. Perineural and vascular invasion have also been identified as having a predictive impact on survival rates. ${ }^{25,26}$ In the present paper, in the post-operative histopathological evaluation of the primary specimens free margins were identified in $71.3 \%$ (62 patients) of them, and $61 \%$ (67 patients) presented with extra-organ invasion, mostly to the larynx. Moreover, perineural and vascular invasion were observed in $52 \%(45 / 87)$ and $56 \%$ (49/87) of tumors respectively. In the present series, positive or close margins and extra-organ spread were also related with decreased survival rates, but with no statistically significant difference ( $35.4 \%$ versus $21.4 \%$; $p>0.05$; and $26.3 \%$ versus $15.5 \% ; p>0.05$ respectively).

Additionally, the regional disease has been identified as the most important prognostic factor in neck tumors, reaffirming the need for accurate staging. ${ }^{25}$ It is well-established in literature that a severe regional disease is associated with poor prognosis, and several authors verified that the extracapsular rupture of the lymph nodes is associated with a higher rate of locoregional recurrence and distant metastasis. ${ }^{26}$ Thus, not only the presence of regional disease, but also the existence of nodal rupture are documented determinant factors in the prognosis. In the present study, the capsular rupture of the lymph nodes, observed in $46 \%$ (68/87), decreased the 5-year DFS, with a statistically significant difference (30.1\% versus $19.8 \%$; $p<0.05$ ).

\section{Conclusion}

The present study confirms the poor prognosis of squamous cell carcinoma of the hypopharynx. Primary PL with neck dissection constitutes a valuable treatment option for locally advanced lesions. Salvage surgical approaches do not seem to improve the survivals rates, and further studies are necessary to understand if salvage PL should be considered among the radical options as an adjuvant treatment strategy after the unsuccessful management of the CRT, or as a palliative approach. 


\section{Financial Support}

This research received no specific grant from any funding agencies, be them commercial or non-profit.

\section{References}

1 Jemal A, Siegel R, Ward E, Murray T, Xu J, Thun MJ. Cancer statistics, 2007. CA Cancer J Clin 2007;57(01):43-66

2 Curado MP, Edwards B, Shin HR, Storm H, Ferlay J, Heanue M, et al. Cancer Incidence in Five Continents, Vol. IX, No. 160. Lyon: IARC; 2007

3 Menvielle G, Fayossé A, Radoï L, et al; ICARE study group. The joint effect of asbestos exposure, tobacco smoking and alcohol drinking on laryngeal cancer risk: evidence from the French populationbased case-control study, ICARE. Occup Environ Med 2016;73 (01):28-33

4 Nair J, Jain P, Chandola U, et al. Gene and miRNA expression changes in squamous cell carcinoma of larynx and hypopharynx. Genes Cancer 2015;6(7-8):328-340

5 Bosetti C, Bertuccio P, Malvezzi M, et al. Cancer mortality in Europe, 2005-2009, and an overview of trends since 1980. Ann Oncol 2013;24(10):2657-2671

6 Bosetti C, Bertuccio P, Levi F, Lucchini F, Negri E, La Vecchia C. Cancer mortality in the European Union, 1970-2003, with a joinpoint analysis. Ann Oncol 2008;19(04):631-640

7 Kuo P, Chen MM, Decker RH, Yarbrough WG, Judson BL. Hypopharyngeal cancer incidence, treatment, and survival: temporal trends in the United States. Laryngoscope 2014;124(09):2064-2069

8 Asakage T, Nakao K, Ebihara Y, Fujishiro Y, Watanabe K. A clinical study of post-cricoid carcinoma. Acta Otolaryngol Suppl 2007; 559(559):118-122

9 Larbcharoensub N, Wattanatranon D, Leopairut J, et al. Clinicopathologic Findings and Treatment Outcome of Laryngectomized Patients with Laryngeal Cancer and Hypopharyngeal Cancer: An Experience in Thailand. Asian Pac J Cancer Prev 2017;18(08): 2035-2042

10 Encinas Vicente A, Souvirón Encabo R, Rodríguez Paramás A, Mancheño Losa M, García de Pedro F, Scola Yurrita B. [Surgical complications in salvage surgery of patients with head and neck carcinomas treated with concomitant chemoradiotherapy (CCR)]. Acta Otorrinolaringol Esp 2007;58(10):454-457

11 Vermorken JB, Specenier P. Optimal treatment for recurrent/ metastatic head and neck cancer. Ann Oncol 2010;21(Suppl 7): vii252-vii261

12 Ferlito A, Shaha AR, Lefebvre JL, Silver CE, Rinaldo A. Organ and voice preservation in advanced laryngeal cancer. Acta Otolaryngol 2002;122(04):438-442

13 Garden AS. Organ preservation for carcinoma of the larynx and hypopharynx. Hematol Oncol Clin North Am 2001;15(02): 243-260, v
14 Hoffman HT, Porter K, Karnell LH, et al. Laryngeal cancer in the United States: changes in demographics, patterns of care, and survival. Laryngoscope 2006;116(9 Pt 2, Suppl 111):1-13

15 Siegel R, Naishadham D, Jemal A. Cancer statistics, 2013. CA Cancer J Clin 2013;63(01):11-30

16 Hall SF, Groome PA, Irish J, O'Sullivan B. The natural history of patients with squamous cell carcinoma of the hypopharynx. Laryngoscope 2008;118(08):1362-1371

17 Pfister DG, Ang KK, Brizel DM, et al; National Comprehensive Concer Network. Head and neck cancers. J Natl Compr Canc Netw 2011;9(06):596-650

18 American Joint Committee on Cancer. Part II. Head and neck sites, pharynx \& larynx. In: Green F, Fritz A, Winchester D, Compton C, Shan J, editors. AJCC cancer staging atlas. 6th ed. New York: Springer Science Business Media; 2002:17-58

19 Kirchner JA. Pyriform sinus cancer: a clinical and laboratory study. Ann Otol Rhinol Laryngol 1975;84(06):793-803

20 Berrino F, Gatta G; EUROCARE Working Group. Variation in survival of patients with head and neck cancer in Europe by the site of origin of the tumours. Eur J Cancer 1998;34(14 Spec No):2154-2161

21 Breda E, Catarino R, Monteiro E. Transoral laser microsurgery as standard approach to hypopharyngeal cancer. Survival analysis in a hospital based population. Acta Otorrinolaringol Esp 2018;69 (01):1-710.1016/j.otorri.2016.11.009

22 Breda E, Catarino R, Monteiro E. Transoral laser microsurgery for laryngeal carcinoma: Survival analysis in a hospital-based population. Head Neck 2015;37(08):1181-1186

23 Goodwin WJ Jr. Salvage surgery for patients with recurrent squamous cell carcinoma of the upper aerodigestive tract: when do the ends justify the means? Laryngoscope 2000;110(3 Pt 2, Suppl 93):1-18

24 López Delgado I, Riestra Ayora J, Arenas Brítez O, García López I, Martínez Guirado T, Scola Yurrita B. Salvage surgery for locoregional recurrences of advanced pharyngolaryngeal squamous cell carcinoma after organ preservation failure. Acta Otorhinolaryngol Ital 2014;34(06):382-388

25 Chirila M, Bolboaca SD, Muresan M, Tomescu E, Cosgarea M. [Lymphatic and vascular invasion in laryngeal and pyriform sinus carcinomas]. Laryngorhinootologie 2011;90(06):358-363

26 Chirilă M, Bolboacă SD, Cosgarea M, Tomescu E, Mureşan M. Perineural invasion of the major and minor nerves in laryngeal and hypopharyngeal cancer. Otolaryngol Head Neck Surg 2009; 140(01):65-69

27 Hoffman HT, Karnell LH, Shah JP, et al. Hypopharyngeal cancer patient care evaluation. Laryngoscope 1997;107(08):1005-1017

28 Johnson CR, Thames HD, Huang DT, Schmidt-Ullrich RK. The tumor volume and clonogen number relationship: tumor control predictions based upon tumor volume estimates derived from computed tomography. Int J Radiat Oncol Biol Phys 1995;33(02): $281-287$ 